\title{
The influence of media reporting of a celebrity suicide on suicidal behavior in patients with a history of depressive disorder
}

\author{
Andrew T.A. Cheng ${ }^{a, *}$, Keith Hawton ${ }^{b}$, Tony H.H. Chen ${ }^{c}$, Amy M.F. Yen ${ }^{c}$, \\ Jung-Chen Chang ${ }^{\mathrm{d}}$, Mian-Yoon Chong ${ }^{\mathrm{e}}$, Chia-Yih Liu ${ }^{\mathrm{f}}$, Yu Lee ${ }^{\mathrm{e}}$, \\ Po-Ren Teng ${ }^{\mathrm{g}}$, Lin-Chen Chen ${ }^{\mathrm{a}}$ \\ a Institute of Biomedical Sciences, Academia Sinica, Taipei, Taiwan \\ ${ }^{\mathrm{b}}$ Centre for Suicide Research, University of Oxford, Warneford Hospital, Oxford, UK \\ ${ }^{\mathrm{c}}$ Institute of Preventive Medicine, College of Public Health, National Taiwan University, Taipei, Taiwan \\ ${ }^{\mathrm{d}}$ Ching Kuo Institute of Management and Health, Keelung, Taiwan \\ e Department of Psychiatry, Chang Gung Memorial Hospital, Kaohsiung, Taiwan \\ ${ }^{\mathrm{f}}$ Department of Psychiatry, Chang Gung Memorial Hospital, Linko, Taiwan \\ ${ }^{g}$ Department of Psychiatry, Show Chwan Memorial Hospital, Changhua, Taiwan
}

Received 5 December 2006; received in revised form 11 January 2007; accepted 12 January 2007

Available online 20 February 2007

\begin{abstract}
Background: Few studies have directly assessed the impact of a specific media report in vulnerable people. This study investigates possible influences of media reporting of a celebrity suicide on subsequent suicidal behaviors and associated risk factors among depressive patients.

Methods: Depressive patients $(N=461)$ were assessed through a structured interview soon after extensive media reporting of a celebrity suicide.

Results: Among 438 depressive patients exposed to the media report, $38.8 \%$ reported an influence on subsequent suicidal behaviors, including $24(5.5 \%)$ with a suicide attempt. The risk of such influence was highest among patients in a severe depressive state just prior to the media report (adjusted OR 7.81, 95\% CI 3.28-18.59). Such influence on a subsequent suicide attempt was highest in patients with a most recent suicide attempt within one month prior to the media reports (adjusted hazard ratio 11.91, 95\% CI 3.76-37.72).

Limitations: Our finding of the significant media influence may reflect adverse thoughts among more suicidal and depressed individuals. The possible influence of other factors on the findings cannot be ruled out.

Conclusions: This study has provided more convincing evidence suggesting negative influences of media reporting of a celebrity suicide on subsequent suicidal behaviors among depressive patients. Particular attention in terms of potential negative media influences should be paid to patients who are younger and currently depressed and have made a recent suicide attempt.
\end{abstract}

(C) 2007 Elsevier B.V. All rights reserved.

* Corresponding author. Tel.: +88622789 9119; fax: +88622782 3047.

E-mail address: bmandrew@gate.sinica.edu.tw (A.T.A. Cheng). 


\section{Introduction}

Certain types of media reports and portrayals of suicide may increase the risk for suicidal behavior, including suicide and attempted suicide (Gould et al., 1988; Schmidtke and Schaller, 2000; Fekete et al., 2001; Hawton and Williams, 2005). The more networks that carry a suicide story, the greater the subsequent increase in suicidal behavior, and glamorous reporting or portrayal of suicide in the media can maximize the risk of 'copycat' suicide (Schmidtke and Hafner, 1988; Gould, 1990; Gould et al., 1994; Yip et al., 2006). However, a major shortcoming of most studies is that exposure to the media report by those who subsequently engaged in suicidal behavior has not been well investigated (Hawton and Williams, 2005).

In this paper, we report findings from a study in which depressive patients were interviewed to assess their exposure to media reporting of a celebrity suicide and its impact on their subsequent suicidal behavior. This study was conducted immediately after the termination of media reporting about a famous television actor in Taiwan, MJ Nee, who committed suicide by hanging from a tree in a countryside fruit yard at the age of 59 years sometime in April, 2005. Extensive media coverage of Nee's suicide started from May 2nd, 2005, the day his body was found, and lasted for 17 days. Aims of the study were to investigate the possible negative influences of media reporting of Nee's suicide on subsequent suicidal behaviors and factors affecting vulnerability to such media influences among depressive patients.

\section{Methods}

\subsection{The media report}

For more than 2 weeks, Nee's suicide appeared on every television news channel and in all newspapers. Five television news channels reported it hourly every day, each report time lasting between minutes and an entire hour as the headline news. News of Nee's death occupied the four most popular national newspapers, with an average coverage of 1.2 (s.d. $=0.7)$ to $2.2($ s.d. $=0.9)$ pages daily.

Detailed examination of the contents of media reports on Nee's suicide showed that all included sensational words and statements of sympathy for the victim. The method used for suicide was repeatedly described in detail, with pictures/photos featuring the tree where he hanged himself, the rope used and his body. The suicide act was depicted as an understandable solution to the victim's dilemma, which included his extramarital relationship and his recent frustrations in show business. Finally, Nee was glorified as a martyr and his past career received adulation (the funeral on May 12th was solemn and majestic, with the President and Vice-President of Taiwan both attending). Several aspects of the guidelines published by the World Health Organization in 2000 for the media regarding how to report on a specific suicide were contravened (www.who.int/mental_health/ media/en/426.pdf) (World Health Organization, 2000). These include: avoidance of sensational reporting, detailed descriptions of the method used, reporting the causes in a simplistic way, especially as a method of coping with personal problems, and glorifying suicide victims as martyrs and objects of public adulation. Also, reports should take account of the impact of suicide on families and other survivors in terms of both stigma and psychological suffering and should include constructive aspects, such as emphasizing that suicide is preventable and depression is treatable. They should also provide information about mental health service resources. There were few examples of constructive aspects of reporting on Nee's suicide.

\subsection{Study subjects and procedure}

Study subjects $(N=461)$ were depressive patients recruited from psychiatric outpatient clinics of six medical centers located in North, Mid, South, and East Taiwan. Six senior staff psychiatrists conducted the research interviews and clinical data collection. All of them received a standard training course on the conduct of a semi-structured clinical interview, the WHO Schedules for Clinical Assessment in Neuropsychiatry (SCAN), with acceptable interrater reliability at both symptom and diagnostic levels (Cheng et al., 2001). Each of them identified the first six patients with a main diagnosis of major depressive disorder according to the Diagnostic and Statistical Manual of Mental Disorders, fourth edition (DSM-IV) (American Psychiatric Association, 1994) from each clinic they held during the month between May 18 and June 17, 2005. Patients with active psychotic or organic symptoms with cognitive impairment were excluded.

The psychiatrists recorded the date of any suicide attempts (and methods used) or suicidal thoughts that respondents said had occurred after the first news report of Nee's death. They also recorded each patient's psychiatric diagnosis and assessed the clinical status of the patient's depression before the first media report based on the following four categories: (1) no depressive illness; (2) in full remission; (3) in partial 
remission; (4) no remission, still with active symptoms. The DSM-IV criteria for full remission (no significant signs or symptoms present during the past two months) and partial remission (symptoms present but full criteria not met, or full remission period less than two months) were used.

The institutional review board of Academia Sinica, Taiwan approved the study, and written informed consent was obtained from the study subjects.

\subsection{Study instrument}

Respondents were asked questions according to a structured questionnaire, which was developed through a process of peer review. The questionnaire was pretested in 10 depressive patients and found to be acceptable with minor linguistic modifications.

The questionnaire first asked about any exposure to the media report of Nee's suicide among respondents. Those who had been exposed to any media reporting of the death were then asked about history and timing of suicidal behavior, including suicidal thoughts or suicide attempts prior to the media reporting. The next question inquired about whether respondents' mood or thoughts had been influenced by such reports, and in what way. Finally, respondents were asked if the media report had influenced any suicidal thoughts or an actual attempt. Respondents' descriptions and explanations about any influence from the media report were recorded verbatim.

\subsection{Statistical analysis}

Logistic regression analysis was used to investigate potential factors which might have increased vulnerability to media influences on suicidal behavior. Univariate analyses were conducted to estimate an odds ratio and 95\% confidence interval for each factor, followed by multivariate analysis to obtain adjusted odds ratios. Trend analysis using likelihood ratio testing was applied to examine whether the risk for suicidal behavior among patients exposed to media reporting increased with the severity of previous suicidal behavior (none, suicidal thought only, and suicide attempt) and the clinical state of depression (full, partial, and no remission) before the news of Nee's death broke. Cox proportional-hazards regression modeling was used to assess the impact of the duration from last suicide attempt to the initial news of Nee's death (May 2nd, 2005) on time to suicide attempt. The survival time was defined as the time period from May 2nd, 2005 to the date of suicide attempt or of interview (for subjects without suicide attempt after the media reports). A $t$-test with Satterthwaite adjustment for unequal variance was also performed to compare the number of attempters in the three-week period before and after May 2nd, 2002. All analyses were conducted using SAS 9.1 (SAS Institute Inc, Cary, NC).

\section{Results}

\subsection{Characteristics of study subjects}

Of 461 depressive patients approached by the investigators, $10(2.2 \% ; 3$ men, 7 women $)$ refused to participate in the study and $13(2.9 \% ; 2$ men, 11 women $)$ had not seen/read any media report about Nee's suicide. Of the remaining 438 respondents $(95 \%)$ with media exposure, $306(69.9 \%)$ were women and the mean (s.d.) age was 42 (15) years and 42 (13) years for men and women, respectively. Overall, $310(70.8 \%)$ had a history of suicidality $(67.4 \%$ of men, $72.2 \%$ of women), including $146(33.3 \%)$ with previous suicide attempt(s) ( $25 \%$ of men, $36.9 \%$ of women). In particular, 82 $(18.7 \%)$ had made suicide attempt(s) in the year before the initiation of media reports on Nee's death $(15.9 \%$ of men, $19.9 \%$ of women), and $138(31.5 \%)$ had persistent suicidal thoughts before being exposed to the reports ( $25.8 \%$ of men, $34.0 \%$ of women).

Among all the patients exposed to the media reporting, $220(50.2 \%)(43.9 \%$ of men, $52.9 \%$ of women) reported suicidality between May $2 \mathrm{nd}$ and the

Table 1

The influence of media reports of a celebrity suicide on suicidal behaviors among 438 depressive patients

\begin{tabular}{llll}
\hline & Male $(N=132) N(\%)$ & Female $(N=306) N(\%)$ & Total $(N=438) N(\%)$ \\
\hline No report of negative influences & $89(67.4)$ & $179(58.5)$ & $268(61.2)$ \\
Report negative influences & $43(32.6)$ & $127(41.5)$ & $170(38.8)$ \\
$\quad$ Suicide thought only & $39(29.5)$ & $107(35.0)$ & $146(33.3)$ \\
$\quad$ Enhanced & 17 & 62 & 79 \\
$\quad$ Occurred first time & 15 & 15 & 22 \\
$\quad$ Recurred & $4(3.0)$ & 30 & 45 \\
Suicide attempt & 2 & $20(6.5)$ & $24(5.5)$ \\
$\quad$ First time & 2 & 5 & 7 \\
$\quad$ Repeat & & 15 & 17 \\
\hline
\end{tabular}


Table 2

Factors affecting vulnerability to negative influences of media report of a celebrity suicide among 438 depressive patients: logistic regression analyses

\begin{tabular}{|c|c|c|c|}
\hline & Affected ${ }^{\mathrm{a}} /$ total $N(\%)$ & Crude odds ratio $(95 \% \mathrm{CI})$ & Adjusted odds ratio $(95 \% \mathrm{CI})$ \\
\hline \multicolumn{4}{|l|}{ Age } \\
\hline$<55 \mathrm{y} / \mathrm{o}$ & $155 / 361(42.9)$ & $1.00^{\mathrm{b}}$ & $1.00^{\mathrm{b}}$ \\
\hline$\geq 55 \mathrm{y} / \mathrm{o}$ & $15 / 77(19.5)$ & $0.32(0.18-0.59)$ & $0.49(0.26-0.94)$ \\
\hline \multicolumn{4}{|l|}{ Gender } \\
\hline Female & $127 / 306(41.5)$ & $1.00^{\mathrm{b}}$ & $1.00^{\mathrm{b}}$ \\
\hline Male & $43 / 132(32.6)$ & $0.68(0.44-1.05)$ & $0.78(0.48-1.25)$ \\
\hline \multicolumn{4}{|l|}{ History of suicidal behavior ${ }^{c}$} \\
\hline None & 24/128 (18.8) & $1.00^{\mathrm{b}}$ & $1.00^{\mathrm{b}}$ \\
\hline Suicide thought only & $66 / 164(40.2)$ & $2.92(1.70-5.02)$ & $2.15(1.21-3.82)$ \\
\hline Suicide attempt & $80 / 146(54.8)$ & $5.25(3.03-9.11)$ & $3.28(1.81-5.93)$ \\
\hline \multicolumn{4}{|l|}{ Previous depressive condition ${ }^{c}$} \\
\hline In full remission/no depression & $7 / 56(12.5)$ & $1.00^{\mathrm{b}}$ & $1.00^{\mathrm{b}}$ \\
\hline In partial remission & $59 / 206(28.6)$ & $3.12(1.34-7.27)$ & $2.97(1.25-7.06)$ \\
\hline No remission & $104 / 176(59.1)$ & $10.94(4.71-25.42)$ & $7.81(3.28-18.59)$ \\
\hline
\end{tabular}

${ }^{\text {a }}$ With negative influences on suicide thoughts and suicide attempts (first and repeat).

b Reference group.

${ }^{c}$ Likelihood ratio test for trend: $P<0.001$ in both univariate and multivariate logistic regression models.

day of interview, with $27(6.2 \%)(3.0 \%$ of men, $7.5 \%$ of women) having made a suicide attempt.

\subsection{The magnitude of media influences}

More than one third (38.8\%) of the 438 respondents exposed to the media reports of Nee's suicide said that this had had a negative influence on their subsequent suicidal thoughts and attempts (Table 1). In 24 (5.5\%) individuals there was a suicide attempt, either for the first time $(1.6 \%)$ or a repeat $(3.9 \%)$. More women than men reported negative effects (41.5\% versus $32.6 \%$ ), but the difference was not statistically significant.

Of 220 respondents with suicidal behavior after the media report, $170(77.3 \%)(74.1 \%$ in men, $78.4 \%$ in women) reported a negative effect from the media report. Of 27 with a suicide attempt, 24 (88.9\%) (100\% in men, $87.0 \%$ in women) reported an effect of the media report. Nearly all of these suicide attempts $(85.2 \%)$ took place in the first 3 weeks following the report. In $6(22.2 \%)$ of these cases with reported media influence ( 2 men and 4 women) the individuals had tried to hang themselves.

\subsection{The content of negative influences}

Several types of explanation were given by respondents for the negative influences of Nee's news on them. The most common descriptions included (a) imitation: "He is a courageous martyr for me to follow elegantly"; "Suicide can be an exit to my suffering from depression like him"; (b) learning how to commit suicide: "My

Table 3

The effect of time from last suicide attempt to the initiation of media report of a celebrity suicide on the risk of suicide attempt: Cox regression analyses

\begin{tabular}{|c|c|c|c|}
\hline & Affected $\mathrm{a} /$ total $N(\%)$ & Crude hazard ratio $(95 \% \mathrm{CI})$ & Adjusted hazard ratio $(95 \% \mathrm{CI})$ \\
\hline \multicolumn{4}{|l|}{ Gender } \\
\hline Female & $20 / 306(6.54)$ & $1.00^{\mathrm{b}}$ & $1.00^{\mathrm{b}}$ \\
\hline Male & $4 / 132(3.03)$ & $0.47(0.16-1.36)$ & $0.50(0.17-1.50)$ \\
\hline \multicolumn{4}{|c|}{ Time from last suicide attempt to May $2 \mathrm{nd}, 2005^{\mathrm{c}}$} \\
\hline No previous attempt & $7 / 292(2.40)$ & $1.00^{\mathrm{b}}$ & $1.00^{\mathrm{b}}$ \\
\hline$<30$ days & $5 / 22(22.72)$ & $11.59(3.67-36.65)$ & $11.91(3.76-37.72)$ \\
\hline $30-90$ days & $5 / 24(20.83)$ & $9.79(3.10-30.89)$ & $8.61(2.76-27.46)$ \\
\hline $90-180$ days & $2 / 14(14.29)$ & $7.21(1.49-34.87)$ & $7.78(1.60-37.81)$ \\
\hline $180-365$ days & $2 / 22(9.09)$ & $3.59(0.74-17.28)$ & $3.38(0.70-16.31)$ \\
\hline$>365$ days & $3 / 64(4.69)$ & $2.03(0.52-7.85)$ & $1.86(0.48-7.24)$ \\
\hline
\end{tabular}

\footnotetext{
${ }^{\text {a }}$ With negative influences on subsequent suicide attempt (first and repeat).

b Reference group.

c Likelihood ratio test for trend: $P<0.001$.
} 
attention has focused on how to tie a rope to hang myself"; (c) rationalization: "A famous big man like him chose suicide to end his life, why not me?"; "His case shows that suicide is not shameful"; "Only after suicide will people care about you"; (d) hopelessness: "Sooner or later my depression will bring me to kill myself like him"; "Doctor's treatment cannot stop him from suicide, perhaps it is useless for me to go on to see my doctor".

\subsection{Factors affecting vulnerability to media influences}

Univariate and multivariate logistic regression analysis (Table 2) indicated that subjects under the age of 55 were more likely to report negative influence of media reports on suicidal behaviors. There was no gender difference in vulnerability to negative media influences. Previous suicidal behavior was a vulnerability factor, with an increasing trend for such an effect from subjects without any history of suicidal behavior to subjects with a history of suicidal thoughts only, and to subjects with a history of suicide attempt $(P<0.001)$.

The same trend was observed for subjects' clinical condition of depression before the initiation of media reports. The risk increased from subjects in full remission or with no history of major depression to subjects in partial remission, and to subjects who were still severely depressed $(P<0.001)$.

\subsection{The effect of time from last suicide attempt}

Table 3 shows the effect of time from the last previous suicide attempt to the initiation of media reports of Nee's suicide on the risk of subsequent suicide attempts which were reported as being influenced by the reports. The lower risk for a suicide attempt in men than in women was not statistically significant. The shorter the time from the last suicide attempt to the start of media reporting the higher the risk of a subsequent suicide attempt. Compared to subjects with no previous suicide attempt, the excess risk of a subsequent suicide attempt declined from 12-fold among those with their last suicide attempt within one month to 8-fold among those within half a year, and further to 2- to 3-fold among those with a time since a previous suicide attempt of longer than half a year. The result of a trend test was statistically significant $(P<0.001)$.

\subsection{How long did the media influences last?}

The total number of subjects with suicide attempts per week in the three-week period after the start of media reports was 23 (mean $=7.67$, s.d. $=0.58)$ com-

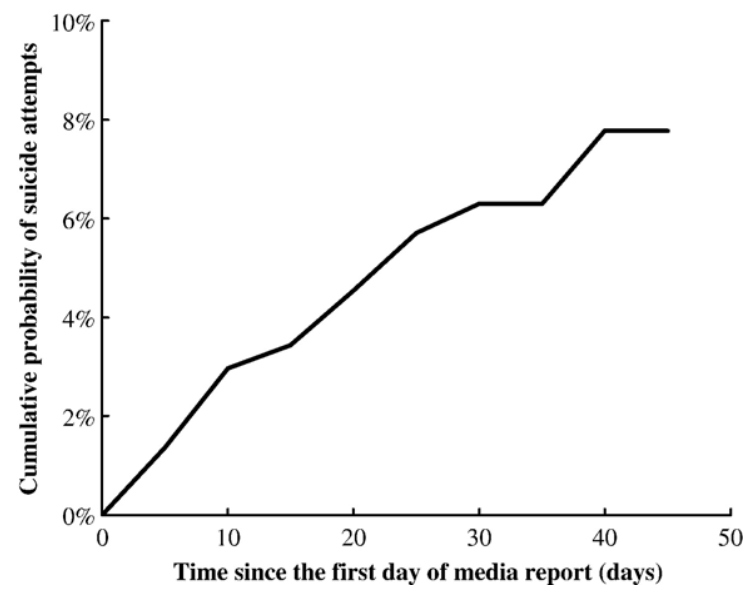

Fig. 1. Cumulative probability of suicide attempts from the first day of media report on a celebrity suicide.

pared with $15($ mean $=5$, s.d. $=0)$ in the same period before the media reports. The mean weekly number was significantly higher following the beginning of media reporting $(t=8.0, P<0.02)$. To assess how long media influences persisted, Fig. 1 shows the cumulative probability of suicide attempts among the respondents after the initiation of the news of Nee's death. The cumulative probability of suicide attempts increased steadily and reached its peak at 40 days with a subsequent leveling off. The log-rank test showed no difference in time to suicide attempt between males and females $\left(\chi^{2}=2.05, P=0.15\right)$.

\section{Discussion}

Improving reporting and portrayal of suicidal behavior in the media has been included in most national suicide prevention strategies (Department of Health and Human Services, 2001; Department of Health, 2002). The evidence to support this comes from studies reporting an increase in suicide and attempted suicide following certain media stimuli (Gould et al., 1988; Schmidtke and Schaller, 2000; Fekete et al., 2001; Hawton and Williams, 2005). There is a need for more evidence that individuals who engage in suicidal behavior following a media event have actually been exposed to it (Hawton and Williams, 2005). The present study has addressed this issue through interviews with patients with major depression, a high risk group for suicide around the globe (Nierenberg et al., 2001; Moller, 2003; Cheng, 1995).

A very high proportion of respondents in this study had been exposed to the media reports of Nee's suicide, and more than one third of the exposed respondents 
reported a negative influence on their subsequent suicidal behavior. Moreover, the negative media influences involved a very large proportion $(88.1 \%)$ of all respondents with suicidal behavior after the media reporting. For $7(29.2 \%)$ of the 24 subjects making a suicide attempt affected by media exposure this was their first-ever attempt. The extremely high rate of exposure was probably due to the very extensive and lengthy media coverage. The large proportion of exposed respondents who indicated negative media influences might be largely attributed to the characteristics of the reports of Nee's suicide. These reports were sensational, repeatedly featuring details of the suicide method, and glorifying the celebrity suicide as a martyr, with adulation over his past career performance. These features have been shown to contribute to media influences on suicidal behavior (Schmidtke and Hafner, 1988; Hawton et al., 1999; Pirkis and Blood, 2001).

The mechanisms involved in the influence of media reporting of suicide on suicidal behavior of those exposed to it are probably complex. Media influences on suicidal behavior are thought to be greater when there is close similarity between the media stimulus or model and the observer, in terms of age, gender, and nationality (Hawton and Williams, 2005), and previous studies have reported a higher vulnerability to media influence among younger people (Gould, 1990). In this study we found a higher risk for negative media influences in people of young age, but not in those of the same sex as the reported celebrity suicide. However, in some studies the suicidal individual appears to have had little in common with the media model, yet apparently identified with him or her sufficiently to trigger suicidal behavior. This is more likely where the model is a celebrity and is revered by the imitator. While modeling of method of suicidal behavior is thought to be an important component of media influence (Hawton and Williams, 2005), we found that few suicide attempters used the same method (hanging) as the model did, probably because the high lethality of this method would have mainly resulted in death by suicide. Finally, social learning theory suggests that the imitator weighs up the extent which the model's behavior is reinforced in deciding whether to imitate it. The comments of some of the participants in this study were in keeping with this.

This study has further identified two important risk factors regarding influence of exposure of suicide in the media. The negative influence on suicidal behaviors was significantly associated with the clinical state of major depression among respondents who were exposed. It was greatest among respondents with active symptoms of major depression prior to the media report with a risk
8 times that among respondents who were in full remission or with no history of such morbidity, and 3 times that among respondents whose major depression was in partial remission. Another risk factor was a previous history of suicidal behavior, with increasing risk from 2-fold among subjects only with previous suicidal thoughts to 3 -fold among those with previous suicide attempt(s).

We have also shown that the negative impact of media influences on subsequent suicide attempts was highest in subjects who made a last suicide attempt within one month prior to the media report. The risk declined the longer the time from the last suicide attempt, but was significant for attempts within 6 months.

Previous studies have assumed that media effects on suicide do not last much beyond two weeks The cumulative probability of suicide attempts over time in this study indicates that the negative influence of the news of Nee's death was probably maintained for 6 weeks from the initiation of the reports, including more than three weeks after the reports ceased. This relatively long effect might be attributed to the unusually long duration of intensive reporting, resulting in a small number of depressive patients with prolonged negative influence.

A number of limitations in this study need to be mentioned. The study relied on self-reports of suicidal behaviors and there was no external verification that these had occurred, nor was there systematic validation of the information about timing of suicidal behavior prior to the media reports. The possible influence of other factors on the findings cannot be ruled out, although the respondents' specific comments on the death of the celebrity make this less likely. Our finding of the significant negative media influence may reflect more negative thoughts among more suicidal and depressed individuals. In other words, depressed patients may be more likely to remember suicidal thoughts and behaviors the more recently they have occurred, so that postreporting incidents are more likely to be reported than pre-reporting incidents. The patients were not asked about the amount of time they spent watching television or reading newspaper reports about Nee's death. Finally, one cannot extrapolate from the findings of this study in patients with depressive disorders to other types of patients or to the community in general.

To our knowledge, this is the first study which has investigated the impact of media report of celebrity suicide on suicidal behaviors using direct interview among depressive patients. The findings provide further convincing evidence to support a strong association between inappropriate media reporting of celebrity suicide and subsequent suicidal behaviors. They support 
the need for more restrained reporting of suicides (Hawton and Williams, 2005). They also suggest that particular attention in terms of potential negative media influences in patients with depressive disorders should be paid to those who are younger, currently depressed, and have made a recent suicide attempt.

\section{Acknowledgements}

We thank the Broadcasting Development Fund, Taiwan, which has kindly provided videotapes of news programs from all television news channels in Taiwan during the three weeks from May 2nd, 2005. This study received no funding.

\section{References}

American Psychiatric Association, 1994. Diagnostic and Statistical Manual of Mental Disorders, 4th edn. APA, Washington, DC. DSM-IV.

Cheng, A.T.A., 1995. Mental illness and suicide: a case-control study in East Taiwan. Arch. Gen. Psychiatry 52, 594-603.

Cheng, A.T.A., Tien, A.Y., Chang, C.J., Cooper, J.E., Brugha, T.S., Lee, C.S., Compton, W., Liu, C.Y., Yu, W.Y., Chen, H.M., 2001. Cross-cultural implementation of a Chinese version of the SCAN in Taiwan. Br. J. Psychiatry 178, 567-572.

Department of Health, 2002. National Suicide Prevention Strategy for England. Department of Health, London.

Department of Health and Human Services, 2001. National Strategy for Suicide Prevention: Goals and Objectives for Action. Department of Health and Human Services, Rockville, MD, U.S.

Fekete, S., Schmidtke, A., Takahashi, Y., Etzersdorfer, E., Upanne, M., Osvath, P., 2001. Mass media, cultural attitudes, and suicide. Results of an international comparative study. Crisis 22, 170-172.
Gould, M.S., 1990. Teenage suicide clusters. JAMA 263, 2051-2052. Gould, M.S., Shaffer, D., Kleinman, M., 1988. The impact of suicide in television movies: replication and commentary. Suicide LifeThreat. Behav. 18, 90-99.

Gould, M.S., Petrie, K., Kleinman, M.H., Wallenstein, S., 1994. Clustering of attempted suicide: New Zealand national data. Int. J. Epidemiol. 23, 1185-1189.

Hawton, K., Williams, K., 2005. Media influences on suicidal behaviour: evidence and prevention. In: Hawton, K. (Ed.), Prevention and Treatment of Suicidal Behaviour: From Science to Practice. Oxford University Press, Oxford, pp. 293-306.

Hawton, K., Simkin, S., Deeks, J.J., O’Connor, S., Keen, A., Altman, D.G., Philo, G., Bulstrode, C., 1999. Effects of a drug overdose in a television drama on presentations to hospital for self-poisoning: time series and questionnaire study. BMJ 318, 972-977.

Moller, H.J., 2003. Suicide, suicidality and suicide prevention in affective disorders. Acta Psychiatr. Scand. 108 (Suppl. 418), 73-80.

Nierenberg, A.A., Gray, S.M., Grandin, L.D., 2001. Mood disorders and suicide. J. Clin. Psychiatry 62 (Suppl. 25), 27-30.

Pirkis, J., Blood, R.W., 2001. Suicide and the media, II: portrayal in fictional media. Crisis 22, 155-162.

Schmidtke, A., Hafner, H., 1988. The Werther effect after television films: new evidence for an old hypothesis. Psychol. Med. 18, 665-676.

Schmidtke, A., Schaller, S., 2000. The role of mass media in suicide prevention. In: Heeringen, K., Hawton, K. (Eds.), The International Handbook of Suicide and Attempted Suicide. John Wiley and Sons, Chichester, England, pp. 675-697.

World Health Organization, 2000. Preventing Suicide: A Resource for Media Professionals. World Health Organization, Geneva.

Yip, P.S., Fu, K.W., Yang, K.C., Ip, B.Y.T., Chan, C.L.W., Chen, E.Y.H., Lee, D.T.S., Law, F.Y.W., Hawton, K., 2006. The effects of a celebrity suicide on suicide rates in Hong Kong. J. Affect. Disord. 93, 245-252. 